\title{
Direct ortho-Arylation of Pyridinecarboxylic Acids: Overcoming the Deactivating Effect of sp2-Nitrogen
}

DOI:

10.1021/acs.orglett.6b03085

\section{Document Version}

Accepted author manuscript

Link to publication record in Manchester Research Explorer

\section{Citation for published version (APA):}

Johnston, A., Ling, K., Sale, D., Lebrasseur, N., \& Larrosa, I. (2016). Direct ortho-Arylation of Pyridinecarboxylic Acids: Overcoming the Deactivating Effect of sp2-Nitrogen. Organic Letters, 18(23), 6094-6097. https://doi.org/10.1021/acs.orglett.6b03085

\section{Published in:}

Organic Letters

\section{Citing this paper}

Please note that where the full-text provided on Manchester Research Explorer is the Author Accepted Manuscript or Proof version this may differ from the final Published version. If citing, it is advised that you check and use the publisher's definitive version.

\section{General rights}

Copyright and moral rights for the publications made accessible in the Research Explorer are retained by the authors and/or other copyright owners and it is a condition of accessing publications that users recognise and abide by the legal requirements associated with these rights.

\section{Takedown policy}

If you believe that this document breaches copyright please refer to the University of Manchester's Takedown Procedures [http://man.ac.uk/04Y6Bo] or contact uml.scholarlycommunications@manchester.ac.uk providing relevant details, so we can investigate your claim.

\section{OPEN ACCESS}




\title{
Direct ortho-Arylation of Pyridinecarboxylic Acids: Overcoming the Deactivating Effect of $\mathbf{s p}^{2}$-Nitrogen
}

\author{
Adam J. S. Johnston, ${ }^{\mathrm{b}}$ Kenneth B. Ling, ${ }^{\mathrm{c}}$ David Sale, ${ }^{\mathrm{c}}$ Nathalie Lebrasseur ${ }^{* \mathrm{~b}}$ and Igor Larrosa ${ }^{* a}$ \\ ${ }^{\text {a }}$ School of Chemistry, University of Manchester, Oxford Road, Manchester, M13 9PL (UK). \\ ${ }^{\mathrm{b}}$ School of Biological and Chemical Sciences, Queen Mary University of London, Mile End Road, London E1 4NS (UK). \\ ${ }^{\mathrm{c}}$ Syngenta, Jealott's Hill International Research Centre, Bracknell, Berkshire, RG42 6EY (UK).
}

Supporting Information Placeholder

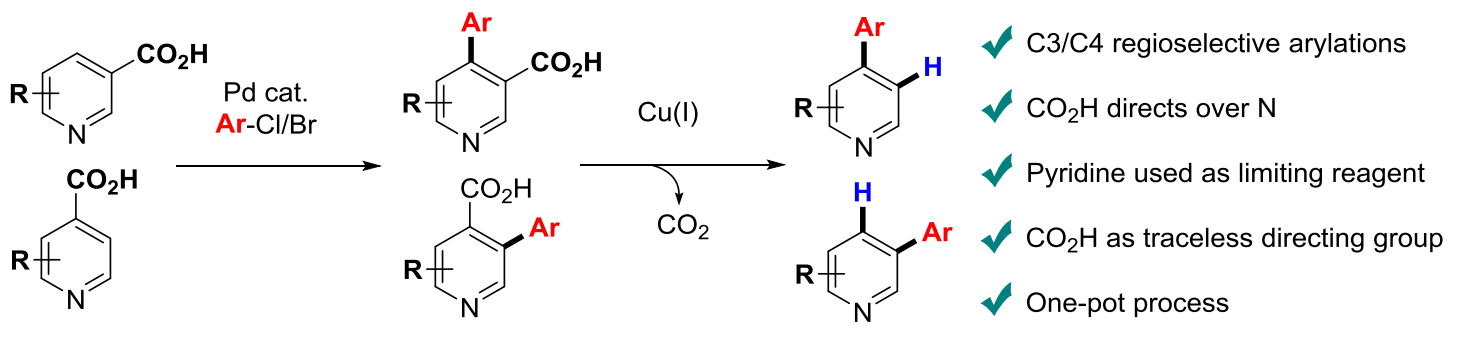

\begin{abstract}
Direct arylations of pyridines are challenging transformations due to the high Lewis basicity of the $\mathrm{sp}^{2}$-nitrogen. Herein we report the use of carboxylates as weakly coordinating directing groups, facilitating the Pd-catalysed C-H arylation of this difficult class of substrates. This methodology allows regioselective $\mathrm{C} 3 / \mathrm{C} 4$ arylation, without the need to use solvent quantities of the pyridine, and using low-cost chloro- and bromoarenes as coupling partners. Furthermore, carboxylates could be employed as traceless directing groups through a one-pot $\mathrm{C}-\mathrm{H}$ arylation / $\mathrm{Cu}(\mathrm{I})$-mediated decarboxylation sequence, thereby accessing directing group free pyridine biaryls.
\end{abstract}

Over the last decade, transition metal-catalysed $\mathrm{C}-\mathrm{H}$ arylation of aromatic and heteroaromatic rings has emerged as an effective method to access functionalized biaryls from simple starting materials. ${ }^{1}$ Key challenges that arise when developing such methodologies lie in the control of the regioselectivity of the reaction and, for less reactive arenes, the need to use them in large excess. ${ }^{2}$ Methodologies targeting arylation of pyridines $^{3,4}$ are of particular interest as these compounds often possess properties of substantial practical utility including their use as ligands for transition metals ${ }^{5}$ and as functional materials, ${ }^{6}$ along with remarkable biological activity. ${ }^{7}$ The challenging nature of the direct arylation of pyridines stems from the high Lewis basicity of the $\mathrm{sp}^{2}$-nitrogen, which often results in catalyst coordination and poisoning and/or in side reactions. Although significant progress has been made towards developing $\mathrm{C} 2$ arylation protocols, ${ }^{3}$ the selective $\mathrm{C}-\mathrm{H}$ arylation of the $\mathrm{C} 3$ and $\mathrm{C} 4$ positions is much less developed. ${ }^{4}$ Common protocols for direct arylation of pyridines require the use of large excess of the pyridine substrate (often as sol vent) ${ }^{[4 \mathrm{~b}, \mathrm{~d}, \mathrm{~g}]}$ or the pre-functionalization of the pyridine core to install activating or directing groups. ${ }^{[4 a, c, e, f]}$ However, such directing groups are often difficult to remove or transform, limiting applicability.

In the last few years, the use of weakly binding carboxylic acids as directing groups has been under intense development. ${ }^{8}$ Their ready availability as starting materials, combined with an array of catalytic methods for their removal or subsequent
Scheme 1. Carboxylic acids as traceless directing groups for $\mathrm{C} 3$ and $\mathrm{C} 4$ regioselective pyridine direct arylation

a) Derivatization of $\mathrm{CO}_{2} \mathrm{H}$ allows pyridine arylation (Yu, 2010 and Su, 2014)

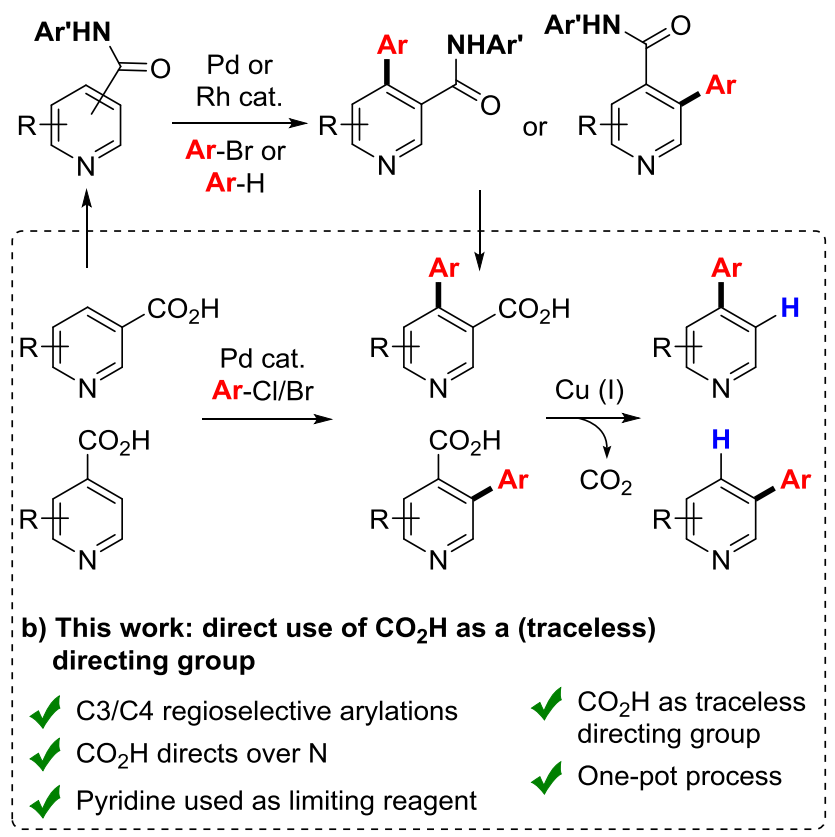


functionalization, ${ }^{9}$ have made carboxylic acids the ideal directing groups for a variety of functionalizations. However, despite its appeal, a methodology able to harness the potential of carboxylic acids for the regioselective $\mathrm{C}-\mathrm{H}$ arylation of pyridines has never been reported. Developing such an approach would require overcoming the strong coordination of pyridine, a common strong directing group for $\mathrm{C}-\mathrm{H}$ arylation, ${ }^{10}$ in favour of the weaker coordination of the carboxylic acid. ${ }^{8 \mathrm{~d}}$ Previous approaches by the groups of $\mathrm{Yu}^{[4 \mathrm{a}]}$ and $\mathrm{Su}^{[4 \mathrm{f}]}$ involved the derivatization of the carboxylic acid into more coordinating amides (Scheme 1a). In this manuscript, we report a Pdcatalyzed system that can directly utilize carboxylic acids as directing groups for arylation at $\mathrm{C} 3$ or $\mathrm{C} 4$ of pyridines in preference to coordination at the pyridine nitrogen (Scheme 1b). Thus, readily available substituted nicotinic acids, used as limiting reagents, can be directly and selectively arylated. Furthermore, the carboxylic acid group can be subsequently removed in a one-pot process, acting as a traceless directing group. ${ }^{11}$

Table 1. Selected optimization results

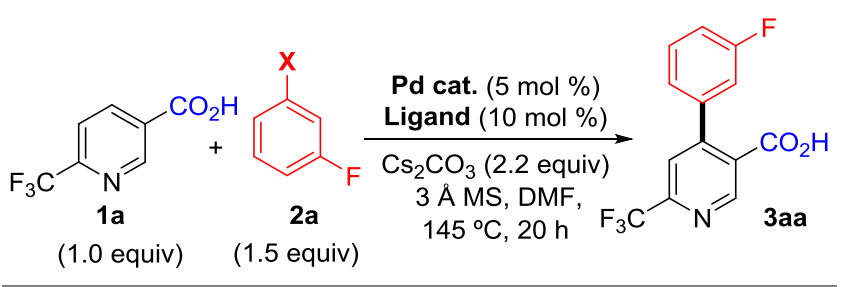

\begin{tabular}{|c|c|c|c|c|c|}
\hline \multirow[t]{2}{*}{ Entry } & \multirow[t]{2}{*}{$X$} & \multirow[t]{2}{*}{ Pd cat. } & \multirow[t]{2}{*}{ Ligand } & \multicolumn{2}{|c|}{$\begin{array}{c}\text { Yield } \\
(\%)\end{array}$} \\
\hline & & & & $1 \mathbf{a}$ & 3aa \\
\hline 1 & $\mathrm{Cl}$ & $\mathrm{Pd}(\mathrm{OAc})_{2}$ & $\operatorname{PAd}_{2}(n-\mathrm{Bu})$ & 19 & 65 \\
\hline 2 & $\mathrm{Cl}$ & $\mathrm{Pd}(\mathrm{OAc})_{2}$ & {$\left[\mathrm{PAd}_{2}(n-\mathrm{Bu}) \mathrm{H}\right] \mathrm{I}$} & 25 & 66 \\
\hline 3 & $\mathrm{Br}$ & $\mathrm{Pd}(\mathrm{OAc})_{2}$ & {$\left[\mathrm{PAd}_{2}(n-\mathrm{Bu}) \mathrm{H}\right] \mathrm{I}$} & 20 & 64 \\
\hline 4 & $\mathrm{Cl}$ & $\begin{array}{c}\text { cataCXium }^{\circledR} \\
\text { A-Pd-G3 }^{b}\end{array}$ & - & 6 & 73 \\
\hline 5 & $\mathrm{Br}$ & $\begin{array}{c}\text { cataCXium }^{\circledR} \\
\text { A-Pd-G3 }^{b}\end{array}$ & - & 26 & 63 \\
\hline
\end{tabular}

${ }^{a}$ Reactions were performed under Ar atmosphere. Yields were determined by ${ }^{1} \mathrm{H}$ NMR analysis using mesitylene or $\mathrm{CH}_{2} \mathrm{Br}_{2}$ as an internal standard.

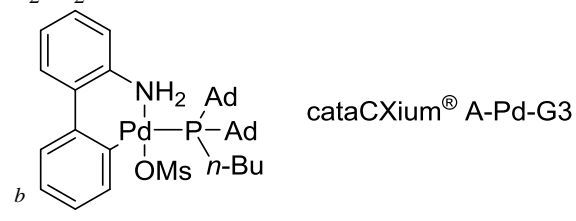

Working on the hypothesis that $N$-coordination is detrimental to any active catalyst species, we choose $\mathbf{1 a}$ as our initial substrate (Table 1) since the large and strongly electronwithdrawing trifluoromethyl group should disfavour such coordination. A variety of C-H arylation methods based either on proposed $\mathrm{Pd}(\mathrm{II}) / \mathrm{Pd}(\mathrm{IV})$ or $\mathrm{Pd}(0) / \mathrm{Pd}(\mathrm{II})$ catalytic cycles were assessed. While the former methods proved inactive, we were delighted to observe that $\mathrm{Pd}(\mathrm{OAc})_{2} / \mathrm{PAd}_{2}(n-\mathrm{Bu})$, a system previously reported by Daugulis et al. for the arylation of benzoic acids, ${ }^{8 a}$ was able to efficiently catalyse the arylation of $\mathbf{1 a}$ with chloroarene $\mathbf{2 a}$ with complete $\mathrm{C} 4$ regioselectivity (entry 1). Remarkably, the pyridine substrate can be the limiting reagent under these conditions. Conveniently, the free phosphine can be replaced by its air stable hydroiodide salt without any drop in yield and selectivity (entry 2), removing the need for the use of a glove box. Along with chloroarenes, bromoarenes are also effective coupling partners under these reaction conditions, thus broadening the scope of this new methodology (entry 3). Further optimisation showed that the yield of 3aa could be increased to $73 \%$ by using the Buchwald $3^{\text {rd }}$ generation precatalyst cataCXium $^{\circledR}$ A-Pd-G3 (entries 4 and 5). ${ }^{12}$

Scheme 2. Scope of the arylation of pyridinecarboxylic acids (1a-n) with chloroarene $2 \mathbf{a}$.

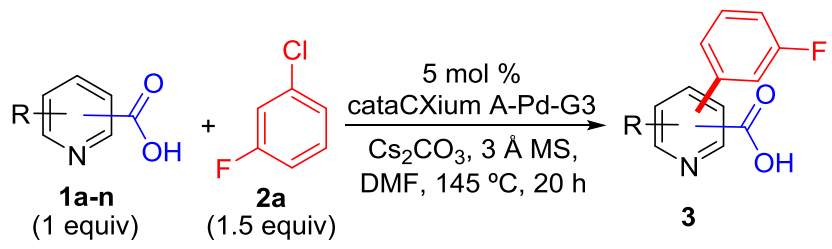

(a) $\mathrm{C} 4$ regioselective arylation<smiles>Cc1nccc(Br)c1C(=O)O</smiles><smiles>Cc1nc(C(F)(F)F)cc(Br)c1C(=O)O</smiles><smiles>Cc1cc(Br)c(C(=O)O)cn1</smiles>

3da: $40 \%$<smiles>O=C(O)c1cnc(OCC(F)(F)F)cc1Br</smiles>

3ga: $43 \%$ $(9: 1 \mathrm{C} 4: \mathrm{C} 2)^{a, c}$<smiles>O=C(O)c1cnccc1Br</smiles>

3ea: $48 \%$ a,c<smiles>O=C(O)c1cnc(N2CCOCC2)cc1Br</smiles><smiles>O=C(O)c1cnncc1Br</smiles>

(b) $\mathrm{C} 3 / \mathrm{C} 5$ regioselective arylation<smiles>Cc1cc(C(=O)O)c(Br)cn1</smiles>

Reactions carried out on a $0.5 \mathrm{mmol}$ scale. Yields are of pure isolated material. ${ }^{a}$ Reactions performed with $5 \mathrm{~mol} \% \mathrm{Pd}(\mathrm{OAc})_{2}$ and $10 \mathrm{~mol} \%\left[\operatorname{PAd}_{2}(n-\mathrm{Bu}) \mathrm{H}\right] \mathrm{I} .{ }^{b}$ After $20 \mathrm{~h} 5 \mathrm{~mol} \% \mathrm{Pd}(\mathrm{OAc})_{2}$, $10 \mathrm{~mol} \%\left[\operatorname{PAd}_{2}(n-\mathrm{Bu}) \mathrm{H}\right] \mathrm{I}$ in $0.5 \mathrm{~mL}$ DMF was added and the reaction stirred for further $20 \mathrm{~h} .{ }^{c}$ The reaction for $48 \mathrm{~h}$.

With optimized conditions in hand the scope of the pyridinecarboxylic acids was next investigated (Scheme 2). We were delighted to find that, in addition to $\mathrm{C} 4$-selective arylation (Scheme 2a, 3aa-3ia), C3/5-arylated pyridines could also be efficiently accessed by employing C2 or C4pyridinecarboxylic acids (Scheme 2b, 3ja-3na). Replacing the 
$\alpha-\mathrm{CF}_{3}$ substituent with a methyl still led to good reactivity (3ca, 3da and 3la). Gratifyingly, both nicotinic (1e) and isonicotinic (1m) acids, despite their poor solubility and lack of $\alpha$ substitution, were also arylated under the reaction conditions (3ea and 3ma respectively) showing that blocking $N$ coordination with an $\alpha$-substituent is not necessary. 4Quinolinecarboxylic acid 1n led to the desired arylated product (3na) in an excellent $83 \%$ yield. Pyridinecarboxylic acids bearing $\alpha$-heteroatoms were also tolerated in this reaction (3faha) although a small amount of the $\alpha$-arylation product was also observed in this case. Pleasingly this protocol could be extended to a pyridazine, albeit in reduced yield (3ia).

Scheme 3. Scope of the arylation of nicotinic acid 1a with haloarenes (2b-n).

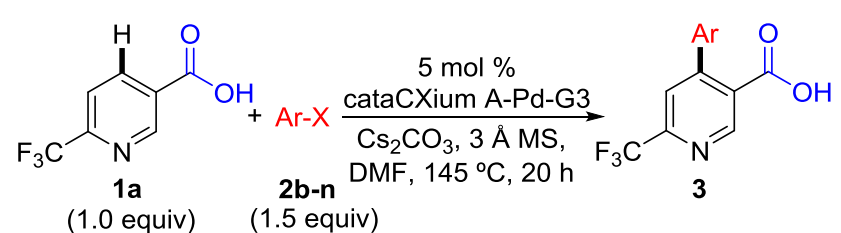<smiles>COc1cccc(Br)c1</smiles>

3ab: $62 \%$ $\mathrm{X}=\mathrm{Cl}$<smiles>Cc1cccc([Al])c1</smiles>

3af: $56 \%$ $\mathrm{X}=\mathrm{Cl}$

$\mathrm{F}$

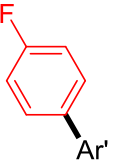

3aj: 53\% $\mathrm{X}=\mathrm{Cl}$<smiles>Cc1cc(C)cc(Br)c1</smiles>

3ac: $62 \%$ $\mathrm{X}=\mathrm{Bra}$<smiles>COc1cc(Br)cc(OC)c1</smiles>

3ag: $38 \%$ Cl

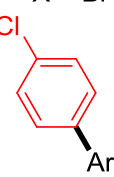

3ak: $55 \%$

$\mathrm{X}=\mathrm{Br}^{\mathrm{a}}$

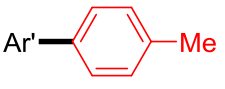

3an: $39 \%$

$\mathrm{X}=\mathrm{Br}^{\mathrm{a}}$

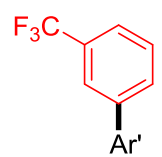

3ad: $71 \%$ $\mathrm{X}=\mathrm{Cl}$

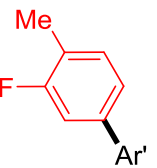

3ah: $51 \%$ $\mathrm{X}=\mathrm{Bra}$<smiles>[Al]c1ccccc1</smiles>

3al: $61 \%$ $\mathrm{X}=\mathrm{Br}^{\mathrm{a}}$

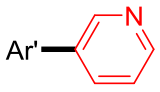

3ao: $20 \%$

$\mathrm{X}=\mathrm{Cl}^{\mathrm{a}}$

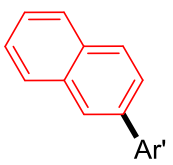

3ae: $58 \%$

$\mathrm{X}=\mathrm{Cl}$

$\mathrm{F}_{3} \mathrm{C}$

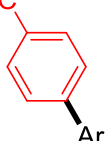

3ai: $68 \%$ $\mathrm{X}=\mathrm{Cl}$

$\mathrm{MeO}$

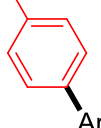

3am: $17 \%$ $\mathrm{X}=\mathrm{Br}^{\mathrm{a}}$
Reactions carried out on a $0.5 \mathrm{mmol}$ scale. Yields are of pure isolated material. a Reactions performed with $5 \mathrm{~mol} \% \mathrm{Pd}(\mathrm{OAc})_{2}$ and $10 \mathrm{~mol} \%\left[\mathrm{PAd}_{2}(n-\mathrm{Bu}) \mathrm{H}\right] \mathrm{I}$.

We next examined the scope of the arylation with respect to the haloarene coupling partner (Scheme 3). Gratifyingly, a variety of substituents could be tolerated in both the meta (3ab-ag) and para (3ag-an) positions, although we found that ortho substituents were not tolerated. Electron-poor or neutral haloarenes generally gave higher yields than electron-rich haloarenes (3am). Even more challenging coupling partners such as 3-chloropyridine (2o) showed reactivity under these conditions, albeit in reduced yield (3ao). When 1-bromo-4chlorobenzene (2k) was used as the coupling partner the reaction showed complete chemoselectivity for arylation of the $\mathrm{C}$ $\mathrm{Br}$ bond leaving the $\mathrm{C}-\mathrm{Cl}$ bond intact for further functionalisation (3ak).
The reaction is amenable to scale up: 1 n reacted with $2 \mathbf{a}$ in a $5 \mathrm{mmol}$ scale without any modifications to the general protocol leading to $0.93 \mathrm{~g}$ of $\mathbf{3 n a}$ in $70 \%$ isolated yield.

Since pyridines are extremely efficient directing groups for ortho arylation of benzenes, ${ }^{10}$ we were interested to examine the regioselectivity of $\mathrm{C}-\mathrm{H}$ arylation when both competing pathways are available (Scheme 4). Accordingly, 6phenylnicotinic acid 10 and 2-phenylisonicotinic acid 1p were tested, as $\mathrm{N}$-directed $\mathrm{C}$-H arylation would occur on the phenyl ring (3'oa and 3'pa) whereas the desired $\mathrm{CO}_{2} \mathrm{H}$-directed arylation would occur on the pyridine ring (3oa and 3pa). Remarkably, the system displayed very high selectivity for arylation

Scheme 4. Regioselectivity in the arylation of phenyl pyridinecarboxylic acids with 2 a.
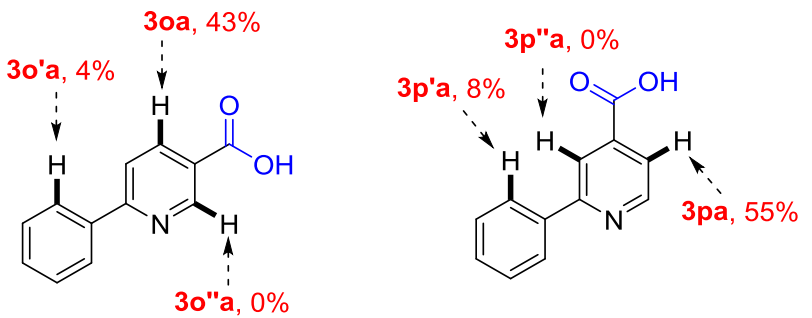

Reaction conditions: $5 \mathrm{~mol} \% \mathrm{Pd}(\mathrm{OAc})_{2}, 10 \mathrm{~mol}_{\%} \mathrm{PAd}_{2}(n-\mathrm{Bu}), 1$ equiv of 10 or $1 \mathbf{p}, 1.5$ equiv $2 \mathbf{a}, 2.2$ equiv $\mathrm{Cs}_{2} \mathrm{CO}_{3}, 3 \mathrm{~A}$ MS, DMF $(0.2 \mathrm{M}), 145^{\circ} \mathrm{C}, 20 \mathrm{~h}$. Yields were determined by ${ }^{1} \mathrm{H}$ NMR analysis of the crude mixture using $\mathrm{CH}_{2} \mathrm{Br}_{2}$ as an internal standard.

Scheme 5. Carboxylic acids as traceless directing groups for C-H arylation of pyridines via a one-pot arylation / protodecarboxylation process.
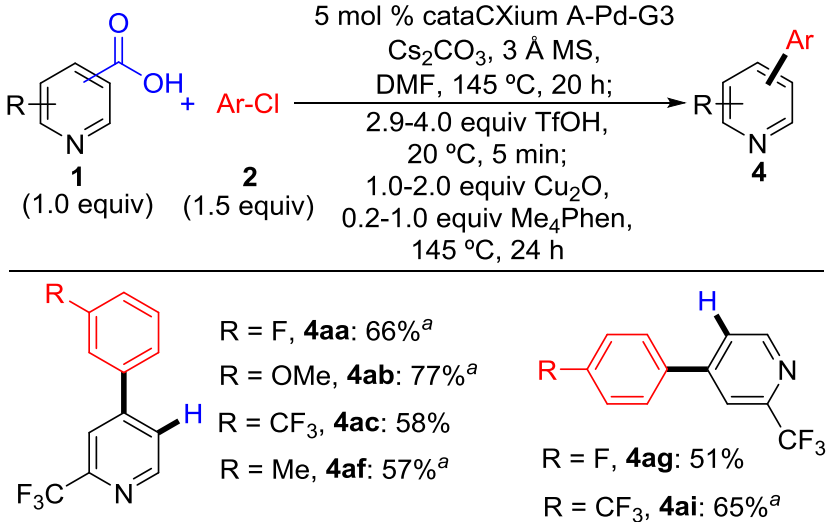<smiles>Cc1cc(Br)cc(C(F)(F)F)n1</smiles><smiles>Cc1cc(Br)ccn1</smiles><smiles>Brc1ccncc1</smiles><smiles>FC(F)(F)c1ccc(Br)cc1</smiles>

4ba: $51 \%$ 4da: $38 \%$ 4ea: $36 \%^{b, c}$

4ja: $63 \%^{b}$<smiles>Brc1cnc2ccccc2c1</smiles><smiles>CC(=[W])c1cccc(F)c1</smiles>

4na: $70 \%^{d}$

Reactions carried out on a $0.5 \mathrm{mmol}$ scale. Yields are of pure isolated material. ${ }^{a}$ 1,10-Phen used instead of $\mathrm{Me}_{4}$ Phen. ${ }^{b}$ Reactions performed with $5 \mathrm{~mol} \% \mathrm{Pd}(\mathrm{OAc})_{2}$ and $10 \mathrm{~mol} \%\left[\mathrm{PAd}_{2}(n-\right.$ $\mathrm{Bu}) \mathrm{H}] \mathrm{I} .{ }^{c} 40 \mathrm{~h}$ arylation time. ${ }^{d} 48 \mathrm{~h}$ decarboxylation time. 
controlled by the weaker $\mathrm{CO}_{2} \mathrm{H}$ directing group with only a small amount of $\mathrm{N}$-directed arylation obtained in both cases.

Having successfully achieved the direct arylation of pyridinecarboxylic acids we next turned our attention towards developing a one-pot arylation / decarboxylation process providing direct access to arylated pyridines. After careful optimization of the reaction conditions, we were pleased to find that arylated pyridines $\mathbf{4}$ could be obtained in good yields via a C-H arylation / copper(I) mediated decarboxylation sequence (Scheme 5). The decarboxylation proceeded equally well at C3 (4aa - 4ea), C2 (4ja) and C4 (4na). To the best of our knowledge, this represents the first strategy for the one-pot formation of directing-group-free, C4-arylated pyridines as single regioisomers.

In conclusion, we have demonstrated that the directing power of carboxylic acids could be successfully harnessed to regioselectively $\mathrm{C}-\mathrm{H}$ arylate pyridines at the $\mathrm{C} 3$ and $\mathrm{C} 4$ positions. Starting from simple pyridinecarboxylic acids and inexpensive chloro- and bromoarenes, a variety of pyridine biaryls could be accessed with high regioselectivity and good yields. Furthermore, the carboxylic acids can be used as traceless directing groups via an efficient one-pot C-H arylation / copper(I)-mediated decarboxylation sequence allowing the formation of directing group free pyridine biaryls.

\section{ASSOCIATED CONTENT}

\section{Supporting Information Available}

Experimental procedures as well as characterization of all previously unknown compounds. The Supporting Information is available free of charge on the ACS Publications website.

\section{Corresponding Author}

*Email: igor.larrosa@manchester.ac.uk

* Email: n.lebrasseur@qmul.ac.uk

\section{ACKNOWLEDGMENT}

We gratefully acknowledge the Engineering and Physical Sciences Research Council and Syngenta for a CASE studentship (A.J.), and the EPSRC National Mass Spectrometry Service (Swansea).

\section{REFERENCES}

1. (a) Alberico, D.; Scott, M. E.; Lautens, M. Chem. Rev. 2007, 107, 174; (b) Giri, R.; Shi, B. F.; Engle, K. M.; Maugel, N.; Yu, J. Q. Chem. Soc. Rev. 2009, 38, 3242; (c) Lyons, T. W.; Sanford, M. S. Chem. Rev. 2010, 110, 1147; (d) Ackermann, L. Chem. Rev. 2011, 111, 1315; (e) Sun, C.-L.; Li, B.-J.; Shi, Z.-J. Chem. Rev. 2011, 111, 1293; (f) Yamaguchi, J.; Yamaguchi, A. D.; Itami, K. Angew. Chem. Int. Ed. 2012, 51, 8960; (g) Wencel-Delord, J.; Glorius, F. Nat. Chem. 2013, 5, 369.

2. (a) Roger, J.; Gottumukkala, A. L.; Doucet, H. ChemCatChem 2010, 2, 20; (b) Nakao, Y. Synthesis 2011, 2011, 3209; (c) Zhao, D.; You, J.; Hu, C. Chem. Eur. J. 2011, 17, 5466; (d) Stephens, D. E.; Larionov, O. V. Tetrahedron 2015, 71, 8683.

3. (a) Seiple, I. B.; Su, S.; Rodriguez, R. a.; Gianatassio, R.; Fujiwara, Y.; Sobel, A. L.; Baran, P. S. J. Am. Chem. Soc. 2010, 132, 13194; (b) Wen, J.; Qin, S.; Ma, L.-F.; Dong, L.; Zhang, J.; Liu, S.-S.; Duan, Y.-S.; Chen, S.-Y.; Hu, C.-W.; Yu, X.-Q. Org. Lett. 2010, 12, 2694; (c) Wang, J.; Wang, S.; Wang, G.; Zhang, J.; Yu, X.-Q. Chem. Commun. 2012, 48, 11769; (d) Liu, B.; Huang, Y.; Lan, J.; Song, F.; You, J. Chem. Sci. 2013, 4, 2163; (e) Patel, N. R.; Flowers, R. A. J. Am. Chem. Soc. 2013, 135, 4672; (f) Singh, P. P.; Aithagani, S. K.; Yadav, M.; Singh, V. P.; Vishwakarma, R. A. J. Org. Chem. 2013, 78, 2639; (g) Ren, X.; Wen, P.; Shi, X.; Wang, Y.; Li, J.; Yang, S.;
Yan, H.; Huang, G. Org. Lett. 2013, 15, 5194; (h) Deb, A.; Manna, S.; Maji, A.; Dutta, U.; Maiti, D. Eur. J. Org. Chem. 2013, 2013, 5251; (i) Xue, D.; Jia, Z.-H.; Zhao, C.-J.; Zhang, Y.-Y.; Wang, C.; Xiao, J. Chem. Eur. J. 2014, 20, 2960; (j) Ma, Z.; Liu, H.; Zhang, C.; Zheng, X.; Yuan, M.; Fu, H.; Li, R.; Chen, H. Adv. Synth. Catal. 2015, 357, 1143; (k) Kan, J.; Huang, S.; Lin, J.; Zhang, M.; Su, W. Angew. Chem. Int. Ed. 2015, 54, 2199.

4. (a) Gürbüz, N.; Özdemir, I.; Çetinkaya, B. Tetrahedron Lett. 2005, 46, 2273; (b) Lafrance, M.; Rowley, C. N.; Woo, T. K.; Fagnou, K. J. Am. Chem. Soc. 2006, 128, 8754; (c) Lafrance, M.; Shore, D.; Fagnou, K. Org. Lett. 2006, 8, 5097; (d) Do, H.-Q.; Daugulis, O. J. Am. Chem. Soc. 2008, 130, 1128; (e) Wasa, M.; Worrell, B. T.; Yu, J.-Q. Angew. Chem. Int. Ed. 2010, 49, 1275; (f) Ye, M.; Gao, G.-L.; Edmunds, A. J. F.; Worthington, P. A.; Morris, J. A.; Yu, J.-Q. J. Am. Chem. Soc. 2011, 133, 19090; (g) Guo, P.; Joo, J. M.; Rakshit, S.; Sames, D. J. Am. Chem. Soc. 2011, 133, 16338; (h) Dai, F.; Gui, Q.; Liu, J.; Yang, Z.; Chen, X.; Guo, R.; Tan, Z. Chem. Commun. 2013, 49, 4634; (i) Aihara, Y.; Chatani, N. Chem. Sci. 2013, 4, 664; (j) Sirois, J. J.; Davis, R.; DeBoef, B. Org. Lett. 2014, 16, 868; (k) Shang, Y.; Jie, X.; Zhao, H.; Hu, P.; Su, W. Org. Lett. 2014, 16, 416; (l) Iaroshenko, V. O.; Gevorgyan, A.; Mkrtchyan, S.; Grigoryan, T.; Movsisyan, E.; Villinger, A.; Langer, P. ChemCatChem 2015, 7, 316; (m) Cambeiro, X. C.; Ahlsten, N.; Larrosa, I. J. Am. Chem. Soc. 2015, 137, 15636; (n) He, Y.; Wu, Z.; Ma, C.; Zhou, X.; Liu, X.; Wang, X.; Huang, G. Adv. Synth. Catal. 2016, 358, 375; (o) Senaweera, S.; Weaver, J. D. J. Am. Chem. Soc. 2016, 138, 2520; (p) Yamada, S.; Murakami, K.; Itami, K. Org. Lett. 2016, 18, 2415; (q) Jiao, J.; Murakami, K.; Itami, K. Chem. Lett. 2016, 45, 529.

5. (a) Graber, S.; Doyle, K.; Neuburger, M.; Housecroft, C. E.; Constable, E. C.; Costa, R. D.; Ortí, E.; Repetto, D.; Bolink, H. J. J. Am. Chem. Soc. 2008, 130, 14944; (b) Wong, W.-Y.; Ho, C.-L. Coord. Chem. Rev. 2009, 253, 1709; (c) Robson, K. C.; Koivisto, B. D.; Berlinguette, C. P. Inorg. Chem. 2012, 51, 1501.

6. (a) Vetrichelvan, M.; Valiyaveettil, S. Chem. Eur. J. 2005, 11, 5889; (b) Oyston, S.; Wang, C.; Perepichka, I. F.; Batsanov, A. S.; Bryce, M. R.; Ahn, J. H.; Petty, M. C. J. Mater. Chem. 2005, 15, 5164.

7. (a) Michael, J. P. Nat. Prod. Rep. 2005, 22, 627; (b) Kassis, P.; Brzeszcz, J.; Bénéteau, V.; Lozach, O.; Meijer, L.; Le Guével, R.; Guillouzo, C.; Lewiński, K.; Bourg, S.; Colliandre, L.; Routier, S.; Mérour, J.-Y. Eur. J. Med. Chem. 2011, 46, 5416; (c) O'Neill, P. M.; Ward, S. A. Angew. Chem. Int. Ed. 2015, 54, 13504; (d) Xie, Y.; Chi, H.-W.; Guan, A.-Y.; Liu, C.-L.; Ma, H.-J.; Cui, D.-L. J. Agric. Food. Chem. 2014, 62, 12491; (e) Xie, Y.; Chi, H.-W.; Guan, A.-Y.; Liu, C.-L.; Ma, H.-J.; Cui, D.-L. Biorg. Med. Chem. 2016, 24, 428; (f) Epp, J. B. et al Biorg. Med. Chem. 2016, 24, 362.

8. (a) Chiong, H. A.; Pham, Q.-N.; Daugulis, O. J. Am. Chem. Soc. 2007, 129, 9879; (b) Engle, K. M.; Mei, T.-S.; Wasa, M.; Yu, J.-Q. Acc. Chem. Res. 2012, 45, 788; (c) Wu, Z.; Chen, S.; Hu, C.; Li, Z.; Xiang, H.; Zhou, X. ChemCatChem 2013, 5, 2839; (d) Liu, Y.-J.; Xu, H.; Kong, W.-J.; Shang, M.; Dai, H.-X.; Yu, J.-Q. Nature 2014, 515 , 389; (e) Zhu, C.; Zhang, Y.; Kan, J.; Zhao, H.; Su, W. Org. Lett. 2015, 17, 3418; (f) Xu, Z.; Yang, T.; Lin, X.; Elliott, J. D.; Ren, F. Tetrahedron Lett. 2015, 56, 475.

9. (a) Gooßen, L. J.; Rodríguez, N.; Linder, C.; Lange, P. P.; Fromm, A. ChemCatChem 2010, 2, 430; (b) Grainger, R.; Nikmal, A.; Cornella, J.; Larrosa, I. Org. Biomol. Chem. 2012, 10, 3172; (d) Dzik, W. I.; Lange, P. P.; Gooßen, L. J. Chem. Sci. 2012, 3, 2671; (e) Grainger, R.; Cornella, J.; Blakemore, D. C.; Larrosa, I.; Campanera, J. M. Chem. Eur. J. 2014, 20, 16680.

10. (a) Ackermann, L.; Vicente, R.; Althammer, A. Org. Lett. 2008, 10, 2299; (b) Kitahara, M.; Umeda, N.; Hirano, K.; Satoh, T.; Miura, M. J. Am. Chem. Soc. 2011, 133, 2160; (c) Li, W.; Yin, Z.; Jiang, X.; Sun, P. J. Org. Chem. 2011, 76, 8543; (d) Zhang, X.; Wang, F.; Qi, Z.; Yu, S.; Li, X. Org. Lett. 2014, 16, 1586.

11. (a) Cornella, J.; Righi, M.; Larrosa, I. Angew. Chem. Int. Ed. 2011, 50, 9429; (b) Luo, J.; Preciado, S.; Larrosa, I. J. Am. Chem. Soc. 2014, 136, 4109; (c) Luo, J.; Preciado, S.; Larrosa, I. Chem. Commun. 2015, 51, 3127.

12. Bruno, N. C.; Tudge, M. T.; Buchwald, S. L. Chem. Sci. 2013, $4,916$. 
